\title{
A special form of Rund's h-curvature tensor using $R 3$-like Finsler space
}

S. T. AVEESH ${ }^{a}$, S. K. NARASIMHAMURTHY ${ }^{a}$, H. G. NAGARAJA ${ }^{b}$, and Pradeep KUMAR ${ }^{a}$

${ }^{a}$ Department of Mathematics, Kuvempu University, Shankaraghatta 577451, Shimoga, Karnataka, India

${ }^{b}$ Department of Mathematics, Bangalore University, Central College Campus, Bangalore 577001, Karnataka, India

E-mails: aveeshst@gmail.com,nmurthysk@gmail.com,nagaraj@bub.ernet.in, pradeepget@gmail.com

\begin{abstract}
The purpose of the present paper is to consider and study a special form of Rund's h-curvature tensor $K_{l j k}^{i}$ and Berwald's curvature tensor $H_{l j k}^{i}$ in an $R 3$-like C-reducible Finsler space. In this paper, we modify the Rund's h-curvature tensor $K_{l j k}^{i}$ to special form by using some special Finsler spaces like C-reducible, R3-like Finsler spaces.
\end{abstract}

2000 MSC: 53C60

\section{Introduction}

Let $F^{n}=\left(M^{n}, F\right)$ be an $n$-dimensional Finsler space with the fundamental function $F(x, y)$. The Finsler $\Gamma_{v}$-connection $R \Gamma$, constructed from the Cartan connection $C \Gamma$, is called the Rund connection. Matsumoto defined the curvature tensor $R \Gamma$ and the concept of special form of Rund's curvature tensor $K_{l j k}^{i}$ [5]. The author [9] have studied Finsler space with Rund's h-curvature tensor $K_{l j k}^{i}$ of a special form. Here we extend the study of a special form of Rund's h-curvature tensor using R3-like Finsler space and obtain some results. In this paper, the range of indexes varies from 1 to $n$ and the $v$-covariant and $o$-covariant derivatives are denoted by $\mid j$ and $\| j$, respectively.

We use the following notations from $[5,8]$ :
(a) $\quad g_{i j}=\frac{1}{2} \dot{\partial}_{i} \dot{\partial}_{j} L^{2}, \quad g^{i j}=\left(g_{i j}\right)^{-1}, \quad \dot{\partial}_{i}=\frac{\partial}{\partial y^{i}}$
(b) $\quad C_{i j k}=\frac{1}{2} \dot{\partial_{k}} g_{i j}, \quad C_{i j}^{k}=\frac{1}{2} g^{k m}\left(\dot{\partial_{m}} g_{i j}\right)$
(c) $h_{i j}=g_{i j}-l_{i} l_{j}, \quad h_{j}^{i}=\delta_{j}^{i}-l^{i} l_{j}$
(d) $\quad C_{l r}^{i} y^{l}=0, \quad h_{l}^{i} y^{l}=0$
(e) $y^{i} L_{k}=y_{k}^{i} L, \quad g_{i j} y^{i} y^{j}=F^{2}, \quad l_{i} y^{i}=F$
(f) $L_{k}=y_{k} L$

Definition 1.1 (see $[1,2])$. A Finsler space $F^{n}(n>3)$ is called $R 3$-like, if the curvature tensor $R_{h i j k}$ is written in the form

$$
R_{h i j k}=g_{h j} L_{i k}+g_{i k} L_{h j}-g_{h k} L_{i j}-g_{i j} L_{h k}
$$

where $L=L_{i j} g^{i j}$ is the tensor. 
Definition 1.2 (see $[2,3]$ ). A Finsler space $F^{n}$ is called C-reducible if it satisfies the equation

$$
C_{i j k}=\left(C_{i} h_{j k}+C_{j} h_{k i}+C_{k} h_{i j}\right) /(n+1)
$$

where $C_{i}=C_{i j k} g^{j k}$.

Definition 1.3 (see [6]). A Finsler space $F^{n}$ is called P-reducible if the torsion tensor $P_{i j k}$ is written as

$$
P_{i j k}=G_{i} h_{j k}+G_{j} h_{k i}+G_{k} h_{i j}
$$

where $P_{i j k}=C_{i j k \mid 0}$ and $G_{i}=C_{i \mid 0} /(n+1)$.

The $v$-covariant derivative of P-reducible Finsler space is given by [9]

$$
P_{j k \mid l}^{i}=G_{\mid l}^{i} h_{j k}+G_{j \mid l} h_{k}^{i}+G_{k \mid l} h_{j}^{i}
$$

Definition 1.4 (see [7]). A Finsler space is called Landsberg Finsler space if $C_{i j k \mid 0}=0$.

We use the following identities from $[5,7,9]$ :
(a) $R_{j k \| l}^{i}-K_{l j k}^{i}+U_{(j k)}\left\{P_{j r}^{i} P_{k l}^{r}+P_{k l \mid j}^{i}\right\}=0$
(b) $R_{l j k}^{i} y^{l}=R_{0 j k}^{i}=R_{j k}^{i}$
(c) $K_{l j k}^{i}+K_{j k l}^{i}+K_{k l j}^{i}=0$
(d) $H_{l j k}^{i}=R_{j k \| l}^{i}$,
(e) $H_{l j k}^{i}+H_{j k l}^{i}+H_{k l j}^{i}=0$,
(f) $\quad K_{l j k}^{i}=-K_{l k j}^{i}, \quad K_{l i j k}=g_{i r} K_{l j k}^{r}$
(g) $h_{i j \| k}=2 C_{i j k}-L^{-2}\left(y_{i} h_{j k}+y_{j} h_{i k}\right)$
(h) $h_{j \| k}^{i}=-L^{-2}\left(y_{j} h_{k}^{i}+y^{i} h_{j k}\right)$

where $R_{j k}^{i}$ is the $(v)$ h-torsion tensor and the suffix ' 0 ' means contraction with $y^{i}$. The notation $u_{(j k)}$ denotes the interchange on indices $j$ and $k$ and substraction.

We use the following lemma in the next section.

Lemma 1.5 (see [4]). If the equation $v_{h i} h_{j k}+v_{i j} h_{h k}+v_{j h} h_{i k}=0$ holds in $F^{n}$, then we have (1) $v_{i j}=0,(n \geq 4)$ and (2) $v_{i j}=v\left(m_{i} n_{j}-m_{j} n_{i}\right)$, with reference to the Moor frame $\left(l^{i}, m^{i}, n^{i}\right)$, where $v$ is a scalar.

\section{Special form of Rund's h-curvature tensor $K_{l j k}^{i}$}

Let $F^{n}$ be a Finsler space with Rund's h-curvature tensor $K_{l j k}^{i}$ of the special form [9]

$$
K_{l j k}^{i}=U_{(j k)}\left\{A_{j k} h_{l}^{i}+D_{l k} h_{j}^{i}+E_{j}^{i} h_{k l}\right\}
$$

where $A_{j k}, D_{l j}, E_{j}^{i}, F_{j}^{i}, G_{l k}$ are Finsler tensor fields.

Consider h-curvature tensor of the form

$$
K_{l j k}^{i}=R_{l j k}^{i}-C_{l r}^{i} R_{j k}^{r}
$$


Using equations (1.2) and (1.3) in (2.2), we get

$$
\begin{aligned}
K_{l j k}^{i}=\{ & \left(\delta_{j}^{i} L_{l k}+g_{l k} L_{j}^{i}-\delta_{k}^{i} L_{l j}-g_{l j} L_{k}^{i}\right) \\
& \left.-\left(\delta_{j}^{r} L_{k}+y_{k} L_{j}^{r}-\delta_{k}^{r} L_{j}-y_{j} L_{k}^{r}\right)\left(C^{i} h_{l r}+C_{l} h_{r}^{i}+C_{r} h_{l}^{i}\right) /(n+1)\right\}
\end{aligned}
$$

By using some Finsler identities, the above equation can be written as

$$
\begin{aligned}
K_{l j k}^{i}=\{ & \left.\left(h_{j}^{i}+l^{i} l_{j}\right) L_{l k}+\left(h_{l k}+l_{l} l_{k}\right) L_{j}^{i}-\left(h_{k}^{i}+l^{i} l_{k}\right) L_{l j}-\left(h_{l j}+l_{l} l_{j}\right) L_{k}^{i}\right\} \\
- & \left\{C^{i} h_{l r} \delta_{j}^{r} L_{k}+C_{l} h_{r}^{i} \delta_{j}^{r} L_{k}+C_{r} h_{l}^{i} \delta_{j}^{r} L_{k}+C^{i} h_{l r} y_{k} L_{j}^{r} C_{l} h_{r}^{i} y_{k} L_{j}^{r}+C_{r} h_{l}^{i} y_{k} L_{j}^{r}\right. \\
& \left.-C^{i} h_{l r} \delta_{k}^{r} L_{j}-C_{l} h_{r}^{i} \delta_{k}^{r} L_{j}-C_{r} h_{l}^{i} \delta_{k}^{r} L_{j}-C^{i} h_{l r} y_{j} L_{k}^{r}-C_{l} h_{r}^{i} y_{j} L_{k}^{r}-C_{r} h_{l}^{i} y_{j} L_{k}^{r}\right\} /(n+1)
\end{aligned}
$$

After simplification and the rearrange the terms, we get

$$
\begin{aligned}
K_{l j k}^{i}= & \left\{h_{j}^{i} L_{l k}+h_{l k} L_{j}^{i}-h_{k}^{i} L_{l j}-h_{l j} L_{k}^{i}\right\}+\left\{l^{i} l_{j} L h_{l k}+l_{l} l_{k} L h_{j}^{i}-l^{i} l_{k} L h_{l j}-l_{l} l_{j} L h_{k}^{i}\right\} \\
& -2\left\{C^{i} h_{l j} L y_{k}+C_{l} h_{j}^{i} L y_{k}+C_{j} h_{l}^{i} L y_{k}-C^{i} h_{l k} L y_{j}-C_{l} h_{k}^{i} L y_{j}-C_{k} h_{l}^{i} L y_{j}\right\} /(n+1) \\
K_{l j k}^{i}= & U_{(j k)}\left[h_{l}^{i}\left(2 C_{k} L y_{j}\right) /(n+1)+h_{j}^{i}\left(L_{l k}+L l_{l} l_{k}-2 C_{l} L y_{k} /(n+1)\right)\right. \\
& \left.\quad+h_{l k}\left(L_{j}^{i}+L l^{i} l_{j}+2 C^{i} L y_{j} /(n+1)\right)\right]
\end{aligned}
$$

In simple form, the above equation can be written as a special form of (2.1) as

$$
K_{l j k}^{i}=U_{(j k)}\left(h_{l}^{i} A_{j k}+h_{j}^{i} D_{l k}+h_{l k} E_{j}^{i}\right)
$$

where

$$
\begin{aligned}
A_{j k} & =2 C_{k} y_{j} L /(n+1) \\
D_{l k} & =\left(L_{l k}+L l_{l} l_{k}-2 C_{l} L y_{k} /(n+1)\right) \\
E_{j}^{i} & =\left(L_{j}^{i}+L l^{i} l_{j}+2 C^{i} L y_{j}\right) /(n+1)
\end{aligned}
$$

Thus we state the following.

Theorem 2.1. In an R3-like, C-reducible Finsler space, the h-curvature tensor reduces to special form of Rund's h-curvature tensor (2.3).

Now we compare the Rund's curvature tensor and h-curvature tensor. Thus, from (2.1) and (2.2), we have

$$
R_{l j k}^{i}-C_{l r}^{i} R_{j k}^{r}=\left\{A_{j k} h_{l}^{i}+D_{l k} h_{j}^{i}+E_{j}^{i} h_{k l}-A_{k j} h_{l}^{i}-D_{l j} h_{k}^{i}-E_{k}^{i} h_{j l}\right\}
$$

Contracting (2.5) with respect to $y^{l}, y^{k}$ and using (1.1d), we get

$$
R_{j 0}^{i}=D_{00} h_{j}^{i}
$$

Again contracting (2.6) with respect to $i$ and $j$, we get

$$
R=(n-1) D_{00}
$$

Now, we will find $D_{00}$. Consider $D_{i j}$ from the special form (2.3), and contract this with respect to $i$ and $j$, and by using (1.1e), we have

$$
D_{00}=2 L F^{2}
$$

Substituting (2.8) in (2.7), we have

$$
R=2(n-1) L F^{2}
$$

Thus we state the following. 
Theorem 2.2. If the Rund's h-curvature tensor has the special form (2.1), then the scalar curvature of the space is $2(n-1) L F^{2}$.

Let us suppose that $F^{n}$ is $R 3$-like C-reducible Finsler space. Then, by using (1.3) and (2.3), the h-curvature tensor (2.2) can written as

$$
\begin{aligned}
R_{l j k}^{i}= & K_{l j k}^{i}+C_{l r}^{i} R_{j k}^{r} \\
R_{l j k}^{i}= & U_{(j k)}\left\{A_{j k} h_{l}^{i}+D_{l k} h_{j}^{i}+E_{j}^{i} h_{k l}\right\} \\
& +\left\{\left(\delta_{j}^{r} L_{k}+y_{k} L_{j}^{r}-\delta_{k}^{r} L j-y_{j} L_{k}^{r}\right)\left(C^{i} h_{l r}+C_{l} h_{r}^{i}+C_{r} h_{l}^{i}\right) /(n+1)\right\} \\
R_{l j k}^{i}= & \left\{A_{j k}+\left(2 C_{j} L y_{k}\right) /(n+1)\right\} h_{l}^{i}-\left\{A_{k j}+\left(2 C_{k} L y_{j}\right) /(n+1)\right\} h_{l}^{i} \\
& +\left\{D_{l k}+2 C_{l} L y_{k} /(n+1)\right\} h_{j}^{i}-\left\{D_{l j}+2 C_{l} L y_{j} /(n+1)\right\} h_{k}^{i} \\
& +\left\{E_{j}^{i}-2 C^{i} L y_{j} /(n+1)\right\} h_{k l}-\left\{E_{k}^{i}-2 C^{i} L y_{k} /(n+1)\right\} h_{j l} \\
R_{l j k}^{i}= & U_{(j k)}\left[\left\{A_{j k}+2 C_{j} L y_{k} /(n+1)\right\} h_{l}^{i}+\left\{D_{l k}+2 C_{l} L y_{k} /(n+1)\right\} h_{j}^{i}\right. \\
& \left.\quad+\left\{E_{j}^{i}-2 C^{i} L y_{j} /(n+1)\right\} h_{k l}\right] \\
R_{l j k}^{i}= & U_{(j k)}\left\{Q_{j k} h_{l}^{i}+N_{l j} h_{k}^{i}+M_{j}^{i} h_{k l}\right\}
\end{aligned}
$$

where

$$
\begin{aligned}
Q_{j k} & =\left\{A_{j k}+2 C_{j} L y_{k} /(n+1)\right\} \\
N_{l k} & =\left\{D_{l k}+2 C_{l} L y_{k} /(n+1)\right\} \\
M_{j}^{i} & =\left\{E_{j}^{i}-2 C^{i} L y_{j} /(n+1)\right\}
\end{aligned}
$$

Thus we have the following.

Theorem 2.3. In an R3-like C-reducible Finsler space, if the Rund's h-curvature tensor has the special form (2.3), then the Cartan h-curvature tensor $R_{l j k}^{i}$ has the special form (2.9).

Using the special form of Rund's h-curvature tensor $K_{l j k}^{i}$ in the Bianchi identity (1.6d), we get

$$
\begin{aligned}
\left(A_{j k}\right. & \left.-A_{k j}+D_{k j}-D_{j k}\right) h_{l}^{i}+\left(A_{k l}-A_{l k}+D_{l k}-D_{k l}\right) h_{j}^{i} \\
& +\left(A_{l j}-A_{j l}+D_{j l}-D_{l j}\right) h_{k}^{i}=0
\end{aligned}
$$

Due to Lemma 1.1, equation (2.10) can be written as

$$
A_{j k}-A_{k j}=D_{j k}-D_{k j}
$$

Thus we have the following.

Theorem 2.4. If the Rund's h-curvature tensor $K_{l j k}^{i}$ is of the special form (2.3), then both the tensor fields $A_{i j}$ and $D_{i j}$ are symmetric simultaneously.

It is also known that a Finsler space is Landsberg space with $P_{i j k}=C_{i j k / 0}=0$. If $F^{n}$ is Landsberg, then from (1.6a) and (1.6e), we get

$$
R_{j k \| l}^{i}-K_{l j k}^{i}=0 \quad \text { or } \quad H_{l j k}^{i}=K_{l j k}^{i}
$$

Thus we can propose the following. 
Corollary 2.5. If $F^{n}$ is a Landsberg space and the Rund's h-curvature tensor $K_{l j k}^{i}$ is of the form (2.3), then Cartan curvature tensor coincides with the Berwald's curvature tensor.

Now consider h-curvature tensor (1.6a) of the form

$$
R_{j k \| l}^{i}=K_{l j k}^{i}-U_{(j k)}\left\{P_{j r}^{i} P_{k l}^{r}+P_{k l \mid j}^{i}\right\}
$$

From equation (1.6e), the above equation can be written as

$$
H_{l j k}^{i}=K_{l j k}^{i}-U_{(j k)}\left\{P_{j r}^{i} P_{k l}^{r}+P_{k l \mid j}^{i}\right\}
$$

Suppose $F^{n}$ is a P-reducible Finsler space, then by using (1.4), (1.5), (2.3), and (2.11), we have

$$
\begin{aligned}
H_{l j k}^{i}= & U_{(j k)}\left\{A_{j k} h_{l}^{i}+D_{l k} h_{j}^{i}+E_{j}^{i} h_{k l}\right\}-U_{(j k)}\left\{P_{j r}^{i} P_{k l}^{r}+P_{k l \mid j}^{i}\right\} \\
H_{l j k}^{i}= & \left\{A_{j k} h_{l}^{i}+D_{l k} h_{j}^{i}+E_{j}^{i} h_{k l}-A_{k j} h_{l}^{i}-D_{l j} h_{k}^{i}-E_{k}^{i} h_{j l}\right\} \\
& -U_{(j k)}\left\{\left(G^{i} h_{j r}+G_{j} h_{r}^{i}+G_{r} h_{j}^{i}\right)\left(G^{r} h_{k l}+G_{k} h_{l}^{r}+G_{l} h_{k}^{r}\right)+\left(G_{k \mid j} h_{l}^{i}+G_{l \mid j} h_{k}^{i}+G_{\mid j}^{i} h_{k l}\right)\right\} \\
H_{l j k}^{i}= & \left\{A_{j k} h_{l}^{i}+D_{l k} h_{j}^{i}+E_{j}^{i} h_{k l}-A_{k j} h_{l}^{i}-D_{l j} h_{k}^{i}-E_{k}^{i} h_{j l}\right\}-\left(G_{k \mid j} h_{l}^{i}+G_{l \mid j} h_{k}^{i}+G_{\mid j}^{i} h_{k l}\right) \\
& +\left(G_{j \mid k} h_{l}^{i}+G_{l \mid k} h_{j}^{i}+G_{\mid k}^{i} h_{j l}\right)-\left\{G^{i} G_{r} h_{j r} h_{k l}+G^{i} G_{k} h_{j l}+G_{j} G^{r} h_{r}^{i} h_{k l}+G_{j} G_{l} h_{k}^{i}\right. \\
& +G_{r} G^{r} h_{j}^{i} h_{k l}+G_{r} G_{k} h_{j}^{i} h_{l}^{r}+G_{r} G_{l} h_{j}^{i} h_{k}^{r}-G^{i} G_{r} h_{k r} h_{j l}-G^{i} G_{j} h_{k l}-G_{k} G^{r} h_{r}^{i} h_{j l} \\
& \left.-G_{k} G_{l} h_{j}^{i}-G_{r} G^{r} h_{k}^{i} h_{j l}-G_{r} G_{j} h_{k}^{i} h_{l}^{r}-G_{r} G_{l} h_{j}^{i} h_{k}^{r}\right\} \\
H_{l j k}^{i}= & U_{(j k)}\left\{T_{j k} h_{l}^{i}+M_{l k} h_{j}^{i}+N_{j}^{i} h_{k l}\right\}
\end{aligned}
$$

where

$$
\begin{aligned}
T_{j k} & =A_{j k}-g_{k \mid j} \\
M_{l k} & =D_{l k}+G_{l \mid k}+G_{k} G_{l}-G_{r} G^{r} h_{k l}-G_{r} G_{k} h_{l}^{r}-G_{r} G_{l} h_{k}^{r} \\
N_{j}^{i} & =E_{j}^{i}-G_{\mid j}^{i}-G^{i} G^{r} h_{j r}-G_{j} G^{r} h_{r}^{i}+G^{i} G_{j}
\end{aligned}
$$

Thus we have the following.

Theorem 2.6. In a P-reducible Finsler space, and the special form of Rund's h-curvature tensor $K_{l j k}^{i}$ has the special form of Berwald's curvature tensor, then $H_{l j k}^{i}$ is of the form (2.12).

Consider the Bianchi identity

$$
H_{l j k}^{i}+H_{j k l}^{i}+H_{k l j}^{i}=0
$$

Substituting (2.12) in (2.13), we get

$$
\begin{aligned}
& {\left[\left(T_{j k}-T_{k j}+M_{k j}-M_{j k}\right) h_{l}^{i}+\left(T_{k l}-T_{l k}+M_{l k}-M_{k l}\right) h_{j}^{i}\right.} \\
& \left.\quad+\left(T_{l j}-T_{j l}+M_{j l}-M_{l j}\right) h_{k}^{i}\right]=0
\end{aligned}
$$

Due to Lemma 1.1, equation (2.14) can be written as

$$
T_{j k}-T_{k j}=M_{j k}-M_{k j}
$$

Thus we have the following. 
Theorem 2.7. If in a Finsler space $F^{n}$ the h-curvature tensor $H_{l j k}^{i}$ is of the form (2.12), then the tensor fields $T_{j k}$ and $M_{k j}$ both are simultaneously symmetric.

\section{Acknowledgement}

The authors are highly thankful to the referee for valuable comments and suggestions.

\section{References}

[1] H. Izumi and T. N. Srivastava. On R3-like Finsler spaces. Tensor (N.S.), 32 (1978), 340-349.

[2] M. Kitayama. Finsler spaces admitting a parallel vector field. Balkan J. Geom. Appl., 3 (1998), $29-36$.

[3] M. Matsumoto. On $C$-reducible Finsler spaces. Tensor (N.S.), 24 (1972), 29-37.

[4] M. Matsumoto. Finsler spaces with $h \gamma$-curvature tensor $P_{h l j k}$ of a special form. Rep. Math. Phys., 14 (1978), 1-13.

[5] M. Matsumoto. Foundations of Finsler Geometry and Special Finsler Spaces. Kaiseisha Press, Otsu, Saikawa, 1986.

[6] S. K. Narasimhamurthy and C. S. Bagewadi. $C$-conformal special Finsler spaces admitting a parallel vector field. Tensor (N.S.), 65 (2004), 162-169.

[7] H. Rund. The Diffrential Geometry of Finsler Spaces. Springer-Verlag, Berlin, 1959.

[8] H. Rund. On Landsberg spaces of scalar curvature. J. Korean Math. Soc., 12 (1959), 79-100.

[9] B. B. Sinha and B. B. Dwivedi. Finsler space with Rund's $h$-curvature tensor $K_{l j k}^{i}$ of a special form. Publ. Inst. Math. (Beograd) (N.S.), 34 (1983), 205-209.

Received September 22, 2008

Revised January 12, 2009 